Correction

\title{
Correction: Weng et al. New International Association for the Study of Lung Cancer (IASLC) Pathology Committee Grading System for the Prognostic Outcome of Advanced Lung Adenocarcinoma. Cancers 2020, 12, 3426
}

\author{
Ching-Fu Weng ${ }^{1,2}$, Chi-Jung Huang ${ }^{3,4,5}$, Shih-Hung Huang ${ }^{6}$, Mei-Hsuan Wu ${ }^{7}$, Ailun Heather Tseng ${ }^{8}$, \\ Yung-Chuan Sung ${ }^{9}$, Henry Hsin-Chung Lee ${ }^{5,10,11, *}$ and Thai-Yen Ling ${ }^{2, *}$
}

check for

updates

Citation: Weng, C.-F.; Huang, C.-J.; Huang, S.-H.; Wu, M.-H.; Tseng, A.H.; Sung, Y.-C.; Lee, H.H.-C.; Ling, T.-Y. Correction: Weng et al. New International Association for the Study of Lung Cancer (IASLC) Pathology Committee Grading System for the Prognostic Outcome of Advanced Lung Adenocarcinoma. Cancers 2020, 12, 3426. Cancers 2021, 13, 4024. https://doi.org/10.3390/ cancers13164024

Received: 22 December 2020

Accepted: 23 June 2021

Published: 10 August 2021

Publisher's Note: MDPI stays neutral with regard to jurisdictional claims in published maps and institutional affiliations.

Copyright: (c) 2021 by the authors. Licensee MDPI, Basel, Switzerland. This article is an open access article distributed under the terms and conditions of the Creative Commons Attribution (CC BY) license (https:// creativecommons.org/licenses/by/ $4.0 /)$.
1 Division of Pulmonary Medicine, Department of Internal Medicine, Hsinchu Cathay General Hospital, Hsinchu 300, Taiwan; cgh18497@cgh.org.tw

2 Department and Graduate Institute of Pharmacology, National Taiwan University, Taipei 100, Taiwan

3 Medical Research Center, Cathay General Hospital, Taipei 106, Taiwan; aaronhuang@cgh.org.tw

Department of Biochemistry, National Defense Medical Center, Taipei 114, Taiwan

School of Medicine, Fu Jen Catholic University, New Taipei 242, Taiwan

6 Division of Pathology, Cathay General Hospital, Taipei 106, Taiwan; ja68@cgh.org.tw

Teaching and Research Center, Hsinchu Cathay General Hospital, Hsinchu 300, Taiwan; markicoo@cgh.org.tw

8 Department of Biomedical Sciences and Engineering, National Central University, Taoyuan 320, Taiwan; c8500@cgh.org.tw

9 Division of Hematology/Oncology, Department of Internal Medicine, Cathay General Hospital, Taipei 106, Taiwan; cgh06496@cgh.org.tw

10 Department of Surgery, Hsinchu Cathay General Hospital, Hsinchu 300, Taiwan

11 Graduate Institute of Translational and Interdisciplinary Medicine, College of Health Sciences and Technology, National Central University, Taoyuan 320, Taiwan

* Correspondence: cgh05589@cgh.org.tw (H.H.-C.L.); tyling@ntu.edu.tw (T.-Y.L.); Tel.: +886-3-527-8999 (ext. 61346) (H.H.-C.L.); +886-2-2312-3456 (ext. 88322) (T.-Y.L.)

The authors would like to make a correction to their published paper [1].

There was a mistake in the original version of the article in Figures 1 and 2. We found that Figures 1 and 2 were misplaced in the article.

They should be replaced with the following Figures 1 and 2:

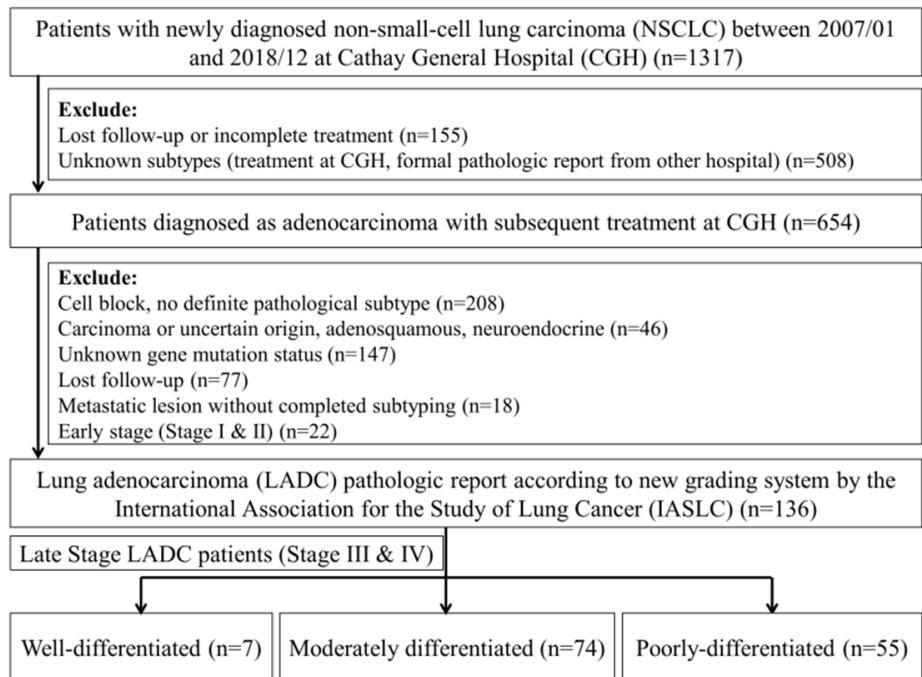

Figure 1. Selection criteria for the subjects. 
(A)

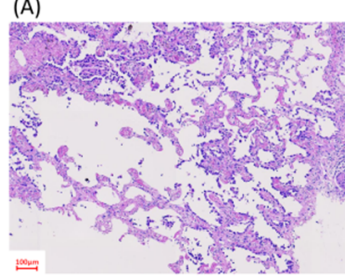

(C)

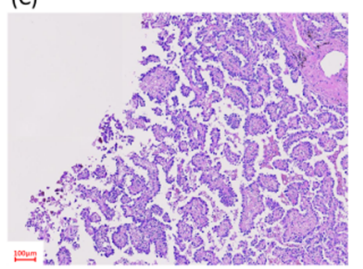

(E)

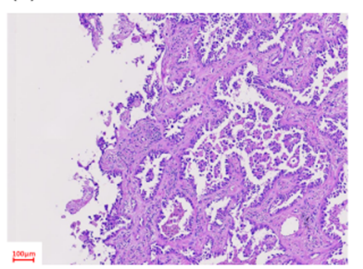

(B)

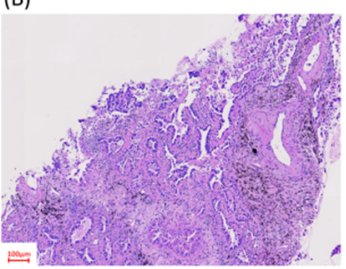

(D)

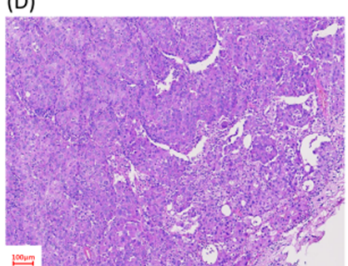

Figure 2. Histological patterns of lung adenocarcinoma. Representative hematoxylin and eosin-stained biopsy specimens: (A) lepidic-predominant pattern; (B) acinar-predominant pattern; (C) papillary-predominant pattern; (D) solid-predominant pattern; and (E) micropapillarypredominant pattern.

The authors apologize for any inconvenience caused and state that the scientific conclusions are unaffected. The original article has been updated.

Funding: This research received no external funding.

Conflicts of Interest: The authors declare no conflict of interest.

\section{Reference}

1. Weng, C.-F.; Huang, C.-J.; Huang, S.-H.; Wu, M.-H.; Tseng, A.H.; Sung, Y.-C.; Lee, H.H.-C.; Ling, T.-Y. New International Association for the Study of Lung Cancer (IASLC) Pathology Committee Grading System for the Prognostic Outcome of Advanced Lung Adenocarcinoma. Cancers 2020, 12, 3426. [CrossRef] [PubMed] 